\title{
IMPACTO DO PLANEJAMENTO DE COMPRAS NO DESEMPENHO FINANCEIRO DA INDÚSTRIA DE TRANSFORMAÇÃO DO BRASIL
}

\author{
Luis Cesar Mondini* \\ cesar.mondini@yahoo.com.br \\ Denise Del Prá Netto Machado* \\ delpra@furb.br \\ Marcia Regina Santiago Scarpin** \\ mrs.scarpin@gmail.com \\ Vanessa Edy Dagnoni Mondini* \\ prof.vanessa@gmail.com \\ *Universidade Regional de Blumenau - Blumenau, SC \\ **Fundação Getúlio Vargas - São Paulo, SP
}

http://dx.doi.org/10.1590/1413-2311.0292014.50270

Recebido em 09/07/2014

Aprovado em 03/02/2015

Disponibilizado em 01/04/2015

Avaliado pelo sistema double blind review

Revista Eletrônica de Administração

Editor: Luís Felipe Nascimento

ISSN 1413-2311 (versão on line)

Editada pela Escola de Administração da Universidade Federal do Rio Grande do Sul.

Periodicidade: Quadrimestral

Sistema requerido: Adobe Acrobat Reader.

\section{RESUMO}

O planejamento estratégico de compras pode ser definido como um processo de avaliação, implementação e controle de importantes decisões de abastecimento usadas para cumprir os planos e metas de longo prazo de uma empresa. Desta maneira, o envolvimento dos profissionais de compras em questões estratégicas é fundamental, pois lhes permite demonstrar que o gerenciamento dos fornecedores tem um impacto direto sobre o desempenho financeiro da empresa. Sob esta perspectiva, o objetivo geral deste estudo é verificar o impacto da estratégia do planejamento de compras no desempenho financeiro da indústria de transformação brasileira. Para isso, foi realizada uma pesquisa quantitativa descritiva, transversal. A amostra foi composta por 312 respondentes que atuam em empresas da indústria de transformação, de diversos ramos de atuação, tanto nacionais como multinacionais. Os dados foram analisados por meio de modelagem de equação estrutural. Como principais resultados, observaram-se os impactos: do Planejamento de Compras sobre o Sistema de Avaliação de Fornecedores e sobre o Relacionamento Comprador-Fornecedor; do Sistema de Avaliação de Fornecedores sobre o Relacionamento Comprador-Fornecedor; e, Relacionamento Comprador-Fornecedor sobre o Desempenho Financeiro percebido. Não foi encontrada significância entre o planejamento de compras e o desempenho financeiro. 
Impacto do planejamento de compras no desempenho financeiro da indústria de transformação do Brasil

Palavras-chave: planejamento de compras; sistema de avaliação de fornecedores; relacionamento comprador-fornecedor.

\title{
THE IMPACT OF SHOPPING PLANNING IN THE FINANCIAL PERFORMANCE OF MANUFACTURING INDUSTRY IN BRAZIL
}

\begin{abstract}
Strategic procurement planning can be defined as a process of evaluation, implementation and control of important decisions of supply used to meet the plans and long-term goals of a company. Thus, the involvement of purchase professionals on strategic issues is critical because it allows them to demonstrate that the management of suppliers have a direct impact on the financial performance of the company. From this perspective, the objective of this study is to assess the impact of procurement planning strategy in the financial performance of the Brazilian manufacturing industry. For this, a descriptive, cross-sectional quantitative survey was conducted. The sample consisted of 312 respondents working in companies in the manufacturing industry, various branches of activity, both domestic and multinationals, and data were analyzed using structural equation modeling. The main results were observed impacts: Planning cart on the Evaluation System Suppliers and the Buyer-Supplier Relationship; the Supplier Evaluation System on the Buyer-Supplier Relationship; and BuyerSupplier Relationships on Financial Performance noticed. Not found any significance between procurement planning and financial performance.
\end{abstract}

Keywords: planning to buy; supplier evaluation system; buyer-supplier relationship.

\section{IMPACTO DE PLANIFICACIÓN PARA COMPRAS EN LA EJECUCIÓN FINANCIERA DE FABRICACIÓN DE BRASIL}

\begin{abstract}
RESUMEN
La planificación de las adquisiciones estratégica puede definirse como un proceso de evaluación, ejecución en el control de las decisiones importantes de la alimentación se utiliza para cumplir con los planes y objetivos a largo plazo de una empresa. Por lo tanto, la participación de los profesionales de las adquisiciones sobre asuntos estratégicos es fundamental, ya que les permite demostrar que la gestión de los proveedores tiene un impacto directo en el desempeño financiero de la empresa. Desde esta perspectiva, el objetivo de este estudio es evaluar el impacto de la estrategia de la planificación de las adquisiciones en el desempeño financiero de la industria manufacturera brasileña. Para esto, se realizó un estudio cuantitativo descriptivo y transversal. La muestra fue compuesta por 312 encuestados que trabajan en empresas de la industria manufacturera, en distintos ramos de la actividad, tanto nacionales como multinacionales, y los datos fueron analizados utilizando modelos de ecuaciones estructurales. En los principales resultados se observaron los impactos: La planificación de los Productores de los Equipos de Evaluación y de la relación compradorproveedor; el Sistema de Evaluación de Proveedores en la relación comprador-proveedor; y relaciones comprador-proveedor en el desempeño financiero notado. No se ha encontrado ninguna significación entre la planificación de las adquisiciones y los resultados financieros.
\end{abstract}


Luis Cesar Mondini, Denise Del Prá Netto Machado, Marcia Regina Santiago Scarpin \& Vanessa Edy Dagnoni Mondini

Palabras clave: planificación de comprar; sistema de evaluación de proveedores; relación comprador-provedor.

\section{INTRODUÇÃO}

O planejamento estratégico de compras pode ser definido como um processo de avaliação, implementação e controle de importantes decisões de abastecimento realizadas para cumprir os planos e metas de longo prazo de uma empresa (CARR; PEARSON, 2002). São previstos no planejamento, a seleção criteriosa de fornecedores, o desenvolvimento de relações de longo prazo, bem como feedbacks contínuos entre compradores e fornecedores (NARASIMHAN; DAS, 2001; TALLURI; NARASIMHAN, 2004). O estreitamento das relações com fornecedores resulta em benefícios aos compradores que passam a receber produtos mais adequados as suas necessidades, exigindo menor rigidez na inspeção e rápida ação corretiva interna ou externa, se preciso (HANSEN; NOHRIA, 2004).

A evolução do relacionamento entre comprador e fornecedor leva os agentes da cadeia produtiva a manterem relações cada vez mais próximas e interdependentes, já que o objetivo comum é a busca por qualidade e competitividade no mercado. Entende-se a capacidade de estabelecer relações de cooperação com os fornecedores como um recurso valioso, único e de difícil imitação. A colaboração pode ser considerada fonte de vantagem competitiva, já que não ocorre automaticamente e exige a necessidade de superação de barreiras e do desenvolvimento de capacidades organizacionais não facilmente copiáveis (HANSEN; NOHRIA, 2004; SU, 2013).

Porém, para que a empresa compradora consiga atingir tal desempenho é preciso elevar o setor de compras a um nível estratégico, que conte com apoio irrestrito dentro da organização, compartilhamento de informações com os principais fornecedores e o desenvolvimento dos fornecedores chave (KOCABASOGLU; SURESH, 2006). O envolvimento dos profissionais de compras em questões estratégicas é fundamental, pois a gestão dos fornecedores, realizada por estes profissionais, impacta diretamente no desempenho financeiro da empresa, em função das negociações sobre prazos de entrega, qualidade do material e tipo de tecnologia utilizada no processo produtivo (CARR; PEARSON, 1999).

Para tanto, o planejamento estratégico de compras precisa desenvolver práticas que auxiliem sua execução. Tais práticas, como a adoção de sistemas de avaliação dos fornecedores, ampliação do relacionamento comprador-fornecedor e desenvolvimento de fornecedores chave, são necessárias para elevar a área de compras a um nível mais estratégico 
Impacto do planejamento de compras no desempenho financeiro da indústria de transformação do Brasil

(CARR; PEARSON, 1999, NARASIMHAN; DAS, 2001). Alinhar estas práticas ao planejamento estratégico de compras auxilia a empresa compradora a atingir os objetivos organizacionais de longo prazo. Empresas compradoras precisam de uma interação próxima com os seus principais parceiros de fornecimento para gerenciar incertezas ambientais. $\mathrm{O}$ desenvolvimento de competências específicas no relacionamento pode levar a vantagens de colaboração para ambas as empresas, neste, que é um mercado dinâmico e competitivo (SU, 2013).

Porém, ainda que as práticas de compras levem a uma vantagem competitiva, poucos são os estudos que as relacionam com desempenho financeiro. Este aspecto abre oportunidades para novas pesquisas, uma vez que é pouco discutido na literatura de operações (CARR; PEARSON, 1999, NARASIMHAN; DAS, 2001). No Brasil, por exemplo, após uma pesquisa nos principais periódicos Qualis A1, A2, B1 e B2, dos últimos 5 anos, foram encontrados 11 artigos sobre tema de compras, dos quais somente 2 relacionavam-se diretamente a questões financeiras (CERIBELI; PRADO, 2011; JORGE et al., 2010).

Com isso, a lacuna teórica que se busca evidenciar neste estudo está relacionada à importância das práticas de compras na melhoria do desempenho financeiro. Para tanto, o estudo utiliza múltiplos respondentes, o que auxilia no desenvolvimento de sua abordagem teórica (NARASIMHAN; DAS, 2001) e foca na indústria brasileira de transformação do Brasil. Segundo Yang et al. (2013), o planejamento de compras é influenciado pela cultura do país em que as empresas estão localizadas, não devendo ser considerada uma teoria universal, e sim, estudos pontuais que possibilitem comparações. A indústria de transformação foi escolhida por se tratar de um setor intermediário, que usa em seu processo produtivo uma diversidade de matérias primas e, portanto, depende de seus fornecedores para manter-se alinhada ao mercado global, por meio da oferta de produtos de qualidade, alta tecnologia e preços competitivos. Além de ser responsável por 13,0\% do PIB brasileiro (IBGE, 2013).

Diante da problemática apresentada, a questão que norteia esse estudo é: Quais práticas do planejamento de compras impactam no desempenho financeiro percebido pelo setor da indústria de transformação brasileira? Com isso, tem-se como objetivo principal, verificar o impacto da estratégia do planejamento de compras no desempenho financeiro da indústria de transformação brasileira.

Este estudo é parte de um projeto mais amplo de pesquisa, e está organizado em sete seções: na próxima, destacam-se a revisão de literatura; na seguinte, descrevem-se os procedimentos metodológicos empregados para a condução da pesquisa; na sequência, 
Luis Cesar Mondini, Denise Del Prá Netto Machado, Marcia Regina Santiago Scarpin \& Vanessa Edy Dagnoni Mondini

apresenta-se a análise dos dados; e, por fim, são expostas as considerações finais e sugestões para futuras pesquisas.

\section{REVISÃO DA LITERATURA}

\subsection{Planejamento de Compras e o Sistema de Avaliação de Fornecedores}

O planejamento estratégico de compras reconhece o relacionamento com os fornecedores como uma prioridade. A partir de sua função estratégia, a empresa consegue estabelecer uma base de fornecimento mais adequada as suas necessidades no dinâmico mercado global (CHIANG et al., 2012). A avaliação de fornecedores torna-se então, uma questão importante que deve ser realizada de forma criteriosa. Neste aspecto, os gestores de compras possuem um papel importante, uma vez que as informações transferidas aos fornecedores sofrem influência de suas percepções e cognições. Este fato pode explicar o porquê de alguns fornecedores melhorarem seu desempenho a partir do resultado das práticas do sistema de avaliação, enquanto outros não conseguem tal feito (HALD; ELLEGAARD, 2011).

Prahinski e Benton (2004) definem o sistema de avaliação do fornecedor como um esforço da empresa compradora em mensurar e melhorar os serviços e/ou produtos recebidos das firmas fornecedoras. $\mathrm{O}$ uso de indicadores para avaliar atividades-meio contribui para a avaliação e o monitoramento da efetividade dos custos, mesmo em uma administração pública (JORGE et al., 2010). Tais programas podem contribuir para a melhoria do desempenho tanto do fornecedor como do comprador, por meio do estabelecimento de uma comunicação eficiente e da troca de conhecimento (PETERSEN et al., 2005). Manter um grupo reduzido de fornecedores também colabora para a eficácia da avaliação, consolidando o volume de compras e contribuindo para a diminuição de custos e melhoria da qualidade (DAS; NARASIMHAN, 2000; NARASIMHAN; DAS, 2001).

Com base em uma revisão de 74 artigos sobre avaliação de fornecedores, Weber et al. (1991) concluíram que na avaliação de fornecedores, a qualidade é o fator mais importante, seguido pelo desempenho de entrega e custos. Martins e Souza (2010) ao analisarem as práticas colaborativas entre camadas, em montadoras de automóveis de Minas Gerais e São Paulo apontaram: (1) a qualidade oferecida, (2) a pontualidade, (3) a rapidez no atendimento, (4) o preço oferecido, (5) a capacidade de produção e, (6) a conjuntura financeira como pontos prioritários utilizados para se avaliar o desempenho dos fornecedores.

REAd | Porto Alegre - Edição 80 - N 1 - janeiro/abril 2015 - p. 113-140 
Impacto do planejamento de compras no desempenho financeiro da indústria de transformação do Brasil

Ainda, segundo os autores, elementos como a participação do fornecedor no resultado final da organização, influência do material adquirido na composição final do produto e adequação à legislação e regulamentações vigentes são critérios levados em conta durante a sistemática de avaliação dos fornecedores.

$\mathrm{Na}$ avaliação dos fornecedores existe uma classificação de grupos com base no desempenho que permite aos gestores de compra a identificação de possíveis candidatos a parcerias estratégicas de longo prazo, programas de desenvolvimento de fornecedores ou ainda, cortes de fornecimento (TALLURI; NARASIMHAN, 2004).

A gestão estratégica do planejamento de compras procura suprir as necessidades dos compradores e da própria organização por meio de processos de suprimentos de longo prazo, com uma seleção, gerenciamento e avaliação sistemática de fornecedores que assegurem a boa qualidade e redução de custos. Além disso, o planejamento de compras influencia a forma como as empresas compradoras identificam seus principais fornecedores e como os programas de avaliação de fornecedores são projetados, implementados e usados (SU, 2013).

H1: O Planejamento de Compras tem um impacto positivo sobre o Sistema de Avaliação de Fornecedores.

\subsection{Planejamento de Compras e o Relacionamento entre Comprador e Fornecedor}

Empresas que consideram o planejamento de compras um fator estratégico, tendem a apreciar as relações de cooperação entre comprador e fornecedor. Neste sentido, os gerentes de compras desempenham um papel crucial no desenvolvimento de relações de trabalho e na comunicação eficaz com os seus fornecedores (CHIANG et al., 2012; CHEN et al., 2004). Diversos estudos estabelecem como crítica a colaboração entre comprador e fornecedor para desempenho operacional da firma (CARR; PEARSON, 1999; CARTER et al., 2000; CHEN et al., 2004). A relação colaborativa entre comprador e fornecedor é um recurso importante que possibilita à empresa compradora acessar, adquirir ou desenvolver novos recursos (SU, 2013). E, ainda que o contrato formal seja importante, Guerra (2014) ressalta que não é suficiente. Em função das restrições de oferta que afetam alguns materiais é importante buscar também o compromisso informal, fomentado pelos laços do bom relacionamento.

A criação, o desenvolvimento e a manutenção de relações de troca entre os parceiros tornaram-se fundamentais para o sucesso de qualquer negócio. Mchug et al. (2003) sugerem algumas características comuns em relações de parceria. Dentre elas, a longevidade do relacionamento no qual a confiança se sobressai à competição; o foco do fornecedor em

REAd | Porto Alegre - Edição 80 - N 1 - janeiro/abril 2015 - p. 113-140 
Luis Cesar Mondini, Denise Del Prá Netto Machado, Marcia Regina Santiago Scarpin \& Vanessa Edy Dagnoni Mondini

qualidade total na prestação dos serviços e entrega dos produtos; e a utilização de cooperação para melhorar o desempenho dos fornecedores (LAIMER; LAIMER, 2009). Relacionamentos baseados em parcerias resultam na aquisição de vantagens competitivas pautadas em variáveis diversas, como a transferência de tecnologia e inovação, redução de custos, melhora no desempenho da entrega e aumento da qualidade.

É por meio da relação entre parceiros que empresas distintas se integram e assumem a responsabilidade pelas diversas etapas do processo produtivo. Práticas colaborativas promovem o estreitamento dos vínculos estabelecidos entre os envolvidos na cadeia e favorecem a coordenação entre as atividades e os processos, garantindo o fluxo contínuo de recursos, imprescindíveis ao desempenho e capacidade de inovação das empresas (MARTINS; SOUZA, 2010).

O desenvolvimento de um relacionamento de longo prazo resulta em ganhos relacionais superiores aos seus participantes, por meio do aumento de criação de valor e da redução dos custos de transação. Conhecida como visão relacional, a vantagem é concebida por meio de investimentos conjuntos, intercâmbio de conhecimentos, combinação de recursos valiosos e escassos e governança eficaz. A atuação conjunta implica em ganhos maiores que não poderiam ser obtidos caso as empresas atuassem de forma isolada (DYER; SINGH,1998).

Entre os mecanismos utilizados por compradores e fornecedores para aumentar o valor derivado de seus relacionamentos, destacam-se a comunicação, o compartilhamento de informações e a confiança (TERPEND et al., 2008). É possível influenciar uma rede de negócios, por exemplo, elevando a qualidade técnica de subcontratados por meio de planejamento e desenvolvimento conjunto de produtos (PEREIRA; LUCE, 2009, p. 17; GUERRA, 2014). Por outro lado, um ineficiente compartilhamento de informações entre os parceiros poderá afetar negativamente a capacidade de flexibilidade de fornecedores que precisam prestar atendimento em circunstâncias imprevistas (MARTINS; SOUZA, 2010). Para Carr e Pearson (1999), empresas que possuem um planejamento estratégico de compras são capazes de promover, em longo prazo, relações de cooperação e comunicação e alcançar uma maior capacidade de resposta às necessidades dos seus fornecedores.

H2: O Planejamento de Compras tem um impacto positivo sobre o Relacionamento entre Comprador e Fornecedor.

\subsection{Planejamento de Compras e o Desempenho Financeiro Percebido}


Impacto do planejamento de compras no desempenho financeiro da indústria de transformação do Brasil

Ellram e Liu (2002) afirmam que o planejamento de compras e o gerenciamento de fornecedores impactam profundamente no desempenho financeiro da empresa. A falta de um planejamento de compras pode incorrer em prejuízos financeiros para as empresas. Merlo, Ceribeli e Prado (2011) constataram que os problemas que originam as perdas de mercadorias em supermercados estão diretamente associados com erros no planejamento e controle.

Chen, Paulraj e Lado (2004) associam a compra estratégica e a gestão de suprimentos à capacidade de resposta ao fornecedor e ao desempenho financeiro da empresa compradora. Para os autores, o planejamento estratégico de compras pode gerar vantagem competitiva sustentável, permitindo às empresas: (a) obter relações de trabalho estreitas com um número limitado de fornecedores; (b) promover a comunicação aberta entre os parceiros da cadeia de suprimentos e; (c) desenvolver relacionamentos estratégicos de longo prazo para obter ganhos mútuos.

Quando a função de compras faz parte do processo de planejamento estratégico, é considerada uma função estratégica de compras. Comprar de forma estratégica requer um entendimento profundo sobre os ativos negociados, sobre as necessidades do comprador e fornecedor e também sobre a natureza da relação entre ambos (MENITA et al., 2011). Muitas empresas reconhecem o valor que a estratégia de compras agrega à empresa. Nestas empresas, a área de compras é pró-ativa e tem a qualidade, habilidades e recursos necessários para realizar operações de nível estratégico (CARR; SMELTZER, 1997). Processos de planejamento bem desenvolvidos e corretamente implantados e controlados contribuem para a obtenção do sucesso no que diz respeito ao desempenho financeiro das empresas (BRACKER et al., 1988).

A capacidade de formar e gerenciar os relacionamentos da cadeia de suprimentos é um ativo organizacional crítico que pode gerar vantagem estratégica durável. As práticas que envolvem o planejamento estratégico contribuem para melhorar a capacidade de resposta aos fornecedores e, conseqüentemente, o desempenho financeiro da empresa compradora (CHEN, PAULRAJ; LADO, 2004). Empresas compradoras que se preocupam em estabelecer uma relação de confiança e uma comunicação efetiva com seus fornecedores possuem efeitos positivos em seu desempenho (CHENG, YIP; YEUNG, 2012).

H3: O Planejamento de Compras tem um impacto positivo sobre o Desempenho Financeiro percebido pela empresa. 
Luis Cesar Mondini, Denise Del Prá Netto Machado, Marcia Regina Santiago Scarpin \& Vanessa Edy Dagnoni Mondini

\subsection{Sistema de Avaliação de Fornecedores e o Relacionamento entre Comprador e Fornecedor}

Os fornecedores representam um recurso fundamental para a empresa. As percepções da empresa compradora com relação ao desempenho atual e esperado de seus fornecedores afetam o seu desempenho direta e indiretamente. A avaliação de fornecedores é um processo de quantificação que está ligado, não só a avaliação do processo de decisão da empresa compradora, mas também, a avaliação do comportamento da empresa fornecedora (HALD; ELLEGAARD, 2011).

Existe uma real necessidade de se avaliar e desenvolver fornecedores já que as organizações não são autossuficientes. Por fazerem parte de cadeias de compradores e fornecedores não há maneira de se tornarem competitivas isoladamente. Segundo Möller et al. (2003), quanto maior for a troca de conhecimentos entre o comprador e o fornecedor, maior será a cooperação na relação. A relação de cooperação consiste em ajudar as empresas a desenvolverem produtos de maior qualidade e assegurar o abastecimento suficiente quando necessário (CARR; PEARSON, 1999).

Além de um bom relacionamento, também é preciso maior compreensão de como os fornecedores trabalham, ressaltando a necessidade de se conhecer substancialmente o seu negócio, entender seus processos de trabalho, respeitar suas capacidades e, por fim, estabelecer um compromisso de prosperidade para com eles. As informações de ambas as partes devem circular abertamente a fim de favorecer que distorções, eventualmente detectadas, sejam corrigidas por meio de um diálogo construtivo. Tanto a segurança desejada pelo comprador, quanto à fidelidade esperada pelo fornecedor, impõem desafios de integração, coordenação e visibilidade entre estes agentes. Estes desafios podem ser sanados por meio da existência de um sistema de avaliação de fornecedores eficiente, que mostre os pontos a serem corrigidos, bem como as necessidades de aprimoramento (LIKER; CHOI, 2004).

A formalização de um sistema de avaliação desta relação (comprador e fornecedor) pode garantir a ambos um feedback do quão eficiente a relação foi, preservando o direito das empresas envolvidas e estabelecendo as fronteiras legais das atividades do processo de desenvolvimento (SOBRERO; ROBERTS, 2001; PRAHINSKI; BENTON, 2004). Um dos benefícios para as empresas que gerenciam ativamente seus fornecedores por meio da utilização de programas formais de avaliação é a eliminação da especulação sobre quais os 
Impacto do planejamento de compras no desempenho financeiro da indústria de transformação do Brasil

fornecedores estão melhores qualificados para justificar o estabelecimento de uma relação mais estreita (CARR; PEARSON, 1999).

A avaliação do fornecedor é uma ferramenta efetiva que permite à empresa compradora melhorar o desempenho e as competências de seus fornecedores. Além disso, é uma forma eficiente de fortalecer as relações com fornecedores por meio de recompensas e do reconhecimento de melhorias, diminuindo o risco de comportamentos oportunistas (SÁNCHEZ-RODRÍGUEZ, 2009; SU, 2013).

H4: O Sistema de Avaliação de Fornecedores tem um impacto positivo sobre o Relacionamento entre Comprador e Fornecedor.

\subsection{Relacionamento entre Comprador e Fornecedor e o Desempenho Financeiro Percebido}

O desenvolvimento de um relacionamento estratégico é de vital importância no fomento e no gerenciamento das competências dos fornecedores. Casos de inovação podem ser induzidos na relação com os compradores, gerando novas competências aos fornecedores (MOREIRA; VARGAS, 2012). Empresas que em suas estratégias priorizam o relacionamento entre os agentes da cadeia, percebem melhoria em seu desempenho geral e no retorno financeiro sobre seu investimento (COHEN; SILVA, 2000). O desempenho financeiro é entendido pelas organizações como o resultado do alcance de suas metas econômicas. Dentre os indicadores mais tradicionais podem ser citados: o crescimento do faturamento e das vendas; o número de novos clientes, mercados e estratégias; o gerenciamento dos custos; o volume de capital de giro; o retorno sobre investimentos e índices de produtividade/eficiência (VENKATRAMAN; RAMANUJAM, 1986).

O desempenho financeiro superior é conseguido quando as empresas focam no desenvolvimento estratégico de seus fornecedores, pois o relacionamento compradorfornecedor estabelece uma cooperação que pode levar ao desenvolvimento de novos produtos, melhorias incrementais e ao compartilhamento de sistemas de informações (SÁNCHEZRODRÍGUEZ, 2009, SOUZA; BACIC; BERNARDES, 2009). No caso de demandas voláteis, um sistema de informação integrado com os principais fornecedores pode trazer benefícios positivos e significativos para o desempenho da empresa compradora, tanto em termos de produtividade, como na rotatividade de estoques (SALDANHA et al., 2013). 
Luis Cesar Mondini, Denise Del Prá Netto Machado, Marcia Regina Santiago Scarpin \& Vanessa Edy Dagnoni Mondini

Estudos como os de Watts e Hahn, (1993), Carr e Pearson, (1999); Chen, Paulraj e Lado, (2004), Cheng, Yip e Yeung, (2012) reforçam que o relacionamento entre comprador e fornecedor tende a levar a empresa a obter melhor desempenho financeiro, pois os preços praticados pelas organizações resultam da capacidade de negociação dos compradores e repercutem nos custos da matéria-prima e de outros insumos (PORTER, 1986). A integração entre fornecedores e compradores é benéfica para ambos. Para os fornecedores, a troca de conhecimentos obtida na relação implica na redução de custos, uma vez que diminui o tempo gasto a procura de maneiras de satisfazer o comprador. E, para os compradores, representa a possibilidade de compartilhar informações dos produtos adquiridos (WATTS; HAHN, 1993; GUERRA, 2014).

As ações de coordenação de compras das empresas com os principais fornecedores podem afetar os seus custos totais (LARSON, 1994). A participação das compras no volume total de gastos das organizações demonstra a importância do investimento em parcerias de longo prazo. Uma vez que os relacionamentos entre compradores e fornecedores se tornam mais cooperativos espera-se que essas empresas também obtenham índices de desempenho financeiro melhores, como maiores retornos sobre o investimento e maiores lucros (CARR; PEARSON, 1999). Para Brito, Brito e Hashiba (2014), a troca de informação e a contenção no uso do poder, possui um impacto positivo no desempenho da empresa. Para os autores, a cooperação com os compradores afeta o crescimento da firma como um todo, enquanto a cooperação com os fornecedores representa maior rentabilidade para a empresa compradora.

H5: O Relacionamento entre Comprador e Fornecedor tem um impacto positivo sobre o Desempenho Financeiro percebido pela empresa.

\section{METODOLOGIA DA PESQUISA}

O principal objetivo deste estudo é verificar o impacto da estratégia do planejamento de compras no desempenho financeiro da indústria de transformação brasileira. O design que caracteriza a presente pesquisa é de natureza descritiva, com um total de 174 empresas analisadas. A perspectiva de estudo foi seccional com avaliação transversal, pois as variáveis de análise focalizadas foram analisadas em um único momento no tempo. A abordagem metodológica foi quantitativa, por meio de levantamento de dados primários, survey com questionário contendo perguntas fechadas. Segundo Hair Jr. et al. (2005), a pesquisa quantitativa é uma investigação empírica cuja finalidade é delinear ou analisar fenômenos, avaliar programas ou isolar variáveis-chave.

REAd | Porto Alegre - Edição 80 - N 1 - janeiro/abril 2015 - p. 113-140 
Impacto do planejamento de compras no desempenho financeiro da indústria de transformação do Brasil

O objeto de estudo deste trabalho foram as empresas pertencentes à indústria de transformação no Brasil, consideradas importantes para a formação do PIB brasileiro. Segundo dados das Contas Nacionais do IBGE, em 2013, a indústria de transformação foi responsável por 13,0\% do PIB. Além disso, estas empresas representam segmentos relevantes para o potencial econômico nacional, podendo ser citados os segmentos: autoindústria (montadoras); alimentício (empresas exportadoras); Higiene e Limpeza (multinacionais); Farmacêutico (multinacionais) entre outros setores.

A população total que a pesquisa abrangeu foi de aproximadamente 1.200 empresas pertencentes ao banco de dados da revista NEI - Noticiário de Equipamentos Industriais, de diversos setores da indústria nacional, tais como: alimentício, automotivos, vidreiros, têxteis, farmacêuticos, siderúrgicos e metalúrgicos, de higiene e limpeza, máquinas e equipamentos, papel e celulose.

A pesquisa foi realizada no período de 05 de janeiro de 2011 até 23 de abril de 2012. As respostas foram coletadas por meio de aplicação de questionários enviados através de $e$ mail diretamente aos respondentes. Porém, algumas empresas mesmo recebendo os questionários por e-mail, foram visitadas posteriormente, a fim de se conseguir um maior número de questionários respondidos. Aproximadamente 190 empresas foram visitadas.

No total foram enviados 1.200 questionários por meio eletrônico. O retorno comprovado pelo servidor de e-mails foram 622 lidos; 36 não lidos; 238 não foram possíveis de ser entregues. Também houve a necessidade de serem reenviados 66 e-mails por ausência temporária, os demais não houve comprovação do procedimento adotado na empresa. Ao final da pesquisa, em 23 de abril 2012, obteve-se o retorno de 218 e-mails, e mais 97 questionários impressos com a pesquisa respondida. Deste total de respondentes conseguiu-se um retorno de 174 empresas do universo de 1.200, sendo que em algumas destas empresas houve mais de um respondente, totalizando 312 respondentes (questionários válidos), seguindo a mesma metodologia adotada pelos autores Carr e Pearson (1999). Observa-se que foram retirados 03 questionários, do total recebido (315) por apresentarem dados faltantes (missing values).

Os dados foram coletados por meio de uma survey utilizando-se como instrumento de coleta um questionário traduzido, com 16 questões fechadas em escala Likert de 5 pontos, aplicado e validado de Carr e Pearson (1999). A tradução do questionário para a língua portuguesa foi realizada considerando as adaptações necessárias para o mercado brasileiro, sem modificar o sentido das perguntas. Foram consideradas as características e apresentadas perguntas no formato de percepção para análise dos respondentes. A validação do REAd | Porto Alegre - Edição 80 - N 1 - janeiro/abril 2015 - p. 113-140 
Luis Cesar Mondini, Denise Del Prá Netto Machado, Marcia Regina Santiago Scarpin \& Vanessa Edy Dagnoni Mondini

questionário foi realizada com o envio dos primeiros 44 questionários por e-mails e mais 20 impressos, totalizando 64 questionários. Após os ajustes necessários e o aval por parte de especialistas, compostos por 3 professores do curso de gestão de operações, e 3 executivos da área de compras, o mesmo foi enviado às empresas pertencentes à amostra.

Os constructos representados por suas variáveis e referencial teórico estão apresentados no Quadro 1.

Quadro 1 - Dimensões Relacionamento Comprador - Fornecedor

\begin{tabular}{|c|c|c|c|}
\hline \multicolumn{2}{|c|}{ Dimensões } & \multirow[b]{2}{*}{$\begin{array}{l}\text { Variáveis } \\
\text { 1. Longo prazo } \\
\text { 2. Mudanças dos planos estratégicos da empresa } \\
\text { 3. Relações (comerciais/financeira/...) com os } \\
\text { fornecedores }\end{array}$} & \multirow[t]{3}{*}{ Autores } \\
\hline D1 & $\begin{array}{c}\text { Planejamento de } \\
\text { Compras }\end{array}$ & & \\
\hline D2 & $\begin{array}{l}\text { Sistema de } \\
\text { Avaliação de } \\
\text { Fornecedores }\end{array}$ & $\begin{array}{l}\text { 4. Certificação de fornecedores } \\
\text { 5. Acompanhamento do desempenho dos fornecedores } \\
\text { 6. Avaliação e reconhecimento do desempenho dos } \\
\text { fornecedores }\end{array}$ & \\
\hline D3 & $\begin{array}{l}\text { Relacionamento } \\
\text { entre } \\
\text { Comprador e } \\
\text { Fornecedor }\end{array}$ & $\begin{array}{l}\text { 7. Acordos especiais com fornecedores } \\
\text { 8. Fidelidade aos principais fornecedores } \\
\text { 9. Reuniões ou encontros com os principais fornecedores } \\
\text { 10. Comunicação direta entre a alta direção/gerências da } \\
\text { empresa e os principais fornecedores } \\
\text { 11. Sistema de troca de dados/informações (EDI) com os } \\
\text { principais fornecedores } \\
\text { 12. Influência na empresa por parte dos principais } \\
\text { fornecedores }\end{array}$ & \multirow[t]{2}{*}{ Carr e Pearson (1999); } \\
\hline D4 & $\begin{array}{l}\text { Desempenho } \\
\text { Financeiro } \\
\text { percebido }\end{array}$ & $\begin{array}{l}\text { 13. Retorno sobre os investimentos da empresa devido ao } \\
\text { relacionamento comprador-fornecedor } \\
\text { 14. Melhora nos lucros das vendas devido à relação } \\
\text { comprador-fornecedor } \\
\text { 15. Melhora do lucro bruto devido à relação comprador- } \\
\text { fornecedor } \\
\text { 16. Evolução do valor presente/PV da empresa nos últimos } \\
\text { cinco anos }\end{array}$ & \\
\hline
\end{tabular}

Fonte: Adaptado de Carr e Pearson (1999)

$\mathrm{Na}$ análise dos dados, optou-se primeiramente por tabulá-los em planilha Microsoft Excel® para serem avaliados e posteriormente importados para o software SPSS® versão 19, para uma análise descritiva e exploratória. Nesta etapa, os itens 10, 11 e 12 da Dimensão 3 foram excluídos por não apresentarem relevância na formação do constructo Relacionamento entre Comprador e Fornecedor.

Após esta primeira fase, considerando o objetivo proposto do estudo partiu-se do modelo de Carr e Pearson (1999) para a obtenção dos índices de ajuste iniciais e garantir sua representatividade junto ao conjunto inteiro de relações causais, com medidas de ajuste absoluto e incremental, por meio da representação pictórica, também chamada de diagrama de caminhos, com setas retilíneas indicando uma relação causal direta de um construto a outro 
Impacto do planejamento de compras no desempenho financeiro da indústria de transformação do Brasil

(HAIR JR. et al., 2005) . Para esse procedimento o software estatístico utilizado foi o SPSS AMOS 18.0, que permite gerar as equações de correlação entre as dimensões, demonstrando graficamente o valor de impacto e os valores para a explicação do modelo $\left(\mathrm{R}^{2}\right)$.

\section{RESULTADO DESCRITIVO DA PESQUISA}

Inicialmente serão descritos o perfil das empresas e dos respondentes, e em seguida as escalas utilizadas no estudo.

\subsection{Perfil das empresas e dos respondentes}

A maioria das empresas atua no mercado nacional, com índice de 45,9\%. Predominantemente essas empresas estão instaladas na região sul, com 24\%. E, 11,9\% desempenham atividades em mais uma região. As regiões norte, nordeste e centro-oeste, são as que possuem o menor número de empresas instaladas. As empresas pesquisadas obtiveram seus segmentos distribuídos de forma equilibrada e os segmentos mais representativos totalizam 66,68\%. Dentre eles, destacam-se o setor alimentício, com a maioria dos respondentes $(14,65 \%)$; o têxtil $(8,08 \%)$; papel e celulose $(7,58 \%)$; siderurgia e metalurgia (7,07\%); autoindústria, fabricantes de máquina, plástico e madeireiro com uma média de 5\% e; higiene e limpeza com uma média de $4 \%$. Estes dez maiores segmentos qualificados pelos respondentes representaram 245 questionários válidos respondidos. Os demais segmentos pesquisados representam 33,32\% constituídos de 67 respondentes, totalizando 312 questionários válidos respondidos.

$\mathrm{O}$ número de funcionários e o faturamento foram indicadores para identificar o tamanho das organizações, 45,40\% das empresas empregam mais de 1.000 funcionários, caracterizando-se como de grande porte, conforme classificação do SEBRAE (2013) e BNDeS - Banco Nacional do Desenvolvimento (2013). Do total de respondentes, 53,2\% pertencem ao departamento de compras, resultado esperado, uma vez que é uma das funções com maior contato com o fornecedor. Seguido do setor de manutenção/almoxarifado - que em muitas empresas representam o setor de compras - com 31,4\%. Em conjunto eles representam $84,6 \%$ do total dos respondentes.

No quesito cargo, a maior parte dos respondentes encontra-se na esfera de auxiliar ou analista, totalizando $64,4 \%$. O questionário foi encaminhado ao responsável do setor de compras, desta forma, este resultado indica que, mesmo sendo este um setor estratégico para

REAd | Porto Alegre - Edição 80 - N 1 - janeiro/abril 2015 - p. 113-140 
Luis Cesar Mondini, Denise Del Prá Netto Machado, Marcia Regina Santiago Scarpin \& Vanessa Edy Dagnoni Mondini

as empresas, as mesmas ainda não despertaram para sua real importância estratégica no desempenho de seus negócios. Ainda segundo os dados, 54,7\% dos entrevistados têm mais de 5 anos de trabalho e $36,7 \%$ possui entre 1 e 5 anos. Estes resultados são importantes, visto que, quando mais tempo de empresa, maior a possibilidade de o respondente conhecer em maior profundidade sua atividade e também a empresa em que atua. Além disso, 68,2\% possuem o ensino superior e $19 \%$ especialização, indicando serem qualificados para o setor que atuam, com nível adequado para o entendimento das perguntas encaminhadas.

\subsection{Escalas}

As escalas apresentadas neste estudo foram validadas e confirmadas por Carr e Pearson (1999) e representam adequadamente seus construtos. Na Tabela 1 é apresentada a matriz de correlações e os dados descritivos de todas as dimensões usadas.

Tabela 1 - Médias, desvios padrões e correlações

\begin{tabular}{|c|c|c|c|c|c|c|}
\hline & Mean & $\mathrm{SD}$ & $\begin{array}{l}\text { Planejamento } \\
\text { de Compras }\end{array}$ & $\begin{array}{l}\text { Sistema de } \\
\text { Avaliação de } \\
\text { Fornecedores }\end{array}$ & $\begin{array}{l}\text { Relacionamento } \\
\text { Comprador } \\
\text { /Fornecedor } \\
\end{array}$ & $\begin{array}{l}\text { Desempenho } \\
\text { Financeiro }\end{array}$ \\
\hline Planejamento de Compras & 4,0 & 0,98 & - & & & \\
\hline $\begin{array}{l}\text { Sistema de Avaliação de } \\
\text { Fornecedores }\end{array}$ & 3,75 & 1,15 & $0,548 * *$ & - & & \\
\hline $\begin{array}{l}\text { Relacionamento } \\
\text { Comprador/Fornecedor }\end{array}$ & 3,81 & 0,76 & $0.519 * *$ & $0,474 * *$ & - & \\
\hline Desempenho Financeiro & 3,77 & 0,89 & $0,450 * *$ & $0,355 * *$ & $0,664 * *$ & - \\
\hline
\end{tabular}

Fonte: Elaborado pelos autores (2013)

A Tabela 1 indica que os resultados demonstram estar dentro da normalidade com relações entre as dimensões trabalhadas com nível de significância $<0.01$.

\section{MEDIDAS DE QUALIDADE/CONFIABILIDADE DO AJUSTE GERAL DO MODELO}

Após analisar as frequências das variáveis do estudo, foi possível verificar que todas as dimensões foram percebidas pelos respondentes da pesquisa. Para auxiliar na confiabilidade da seleção das dimensões, foi calculado o alfa de Cronbach. Esse índice, segundo Hair Jr. et al. (2005), indica o grau de convergência das respostas; assim, quanto 
Impacto do planejamento de compras no desempenho financeiro da indústria de transformação do Brasil

maior o índice, maior a fidedignidade delas. Ainda segundo o autor, índices superiores a 0,7 são considerados satisfatórios. Os resultados estão representados no Quadro 2.

Quadro 2 - Dimensões representativas no Desempenho Financeiro percebido na relação comprador e fornecedor

\begin{tabular}{|l|l|l|l|}
\hline \multicolumn{2}{|l|}{ Dimensões } & Percepção & Alfa de Cronbach \\
\hline D1 & Planejamento de Compras & Sim & 0,835 \\
\hline D2 & Sistema de Avaliação de Fornecedores & Sim & 0,763 \\
\hline D3 & Relacionamento entre Comprador e Fornecedor & Sim & 0,899 \\
\hline D4 & Desempenho Financeiro percebido. & Sim & 0,736 \\
\hline
\end{tabular}

Fonte: Elaborado pelos autores (2013)

\subsection{Ajuste Geral do Modelo}

A partir da adequação no modelo original, medidas de ajuste absoluto foram empregadas nas dimensões selecionadas, tais como Qui-quadrado da razão de verossimilhança $\left(\chi^{2}\right)$ e a raiz do resíduo quadrático médio, com a finalidade de certificar a representação adequada do conjunto inteiro de relações causais, apresentados na Tabela 2.

Tabela 2 - Estatística Qui-quadrado $\left(\chi^{2}\right)$

\begin{tabular}{l|l}
\hline Qui-quadrado $\left(\chi^{2}\right)$ & 89,990 \\
\hline Grau de liberdade & 39 \\
\hline Probability level &, 000 \\
\hline$\chi^{2} / \mathrm{GL}$ & 2,307 \\
\hline RMSEA & 0,065 \\
\hline
\end{tabular}

Fonte: Elaborado pelos autores (2013)

$\mathrm{Na}$ Tabela 2 o que se busca é um valor de Qui-quadrado $\left(\chi^{2}\right)$ não significativo, dado que H0 indica que os dados se ajustam ao modelo. No caso de significativo, Hair Jr. et al. (2005) defendem que se possa dividir o seu valor pelos graus de liberdade $\left(\chi^{2} / \mathrm{GL}\right)$, sendo valores iguais ou inferiores a 5, aceitáveis.

Complementando o Qui-quadrado $\left(\chi^{2}\right)$, foi realizada a raiz do erro quadrático de aproximação (RMSEA), que resultou em um valor de 0,065 , representando qualidade no ajuste do modelo, visto que os valores recomendados variam entre 0,05 a 0,08 (HAIR Jr. et al, 2005). Além das medidas apresentadas, também foram realizadas outras medidas de ajuste incremental. Pela Tabela 3 é possível verificar os indicadores que representam tais medidas.

Tabela 3 - Medidas de Ajuste incremental

\begin{tabular}{l|l|l}
\hline Tipo de medida & $\begin{array}{l}\text { Valor } \\
\text { modelo }\end{array}$ & $\begin{array}{l}\text { Valor de referência } \\
\text { (HAIR Jr. et al, 2005) }\end{array}$ \\
\hline Normed Fit Index (NFI) & 960 &, 90 \\
\hline Tucker-Lewis Index ou Non-Normed Fit Index (NNFI) &, 967 &, 90 \\
\hline Comparative Fit Index (CFI) Fonte: Elaborado pelos autores (2013)
\end{tabular}


Luis Cesar Mondini, Denise Del Prá Netto Machado, Marcia Regina Santiago Scarpin \& Vanessa Edy Dagnoni Mondini

Existem diversas outras medidas de ajuste, porém foram selecionadas as principais, como forma de demonstrar que os níveis de qualidade de ajustes estão adequados, com valores acima de 0,90 .

Além das medidas de ajuste calculou-se ainda o índice Variância Extraída Média AVE (Average Variance Extracted). O índice AVE reflete a percentagem de variância dos itens que é capturada pelo respectivo fator, seu valor deverá se superior a 0,50 para garantir que o fator contribui significativamente para seus indicadores (FORNELL; LARCKER, 1981). Os resultados foram: (0,83) Planejamento de Compras; $(0,87)$ Sistema de Avaliação de Fornecedores; (0,55) Relacionamento comprador-fornecedor e; $(0,80)$ Desempenho Financeiro. Também foi realizado o teste de variance inflation factor (VIF) para observar a multicolinearidade entre os construtos. Os resultados não apresentaram problemas de multicolinearidade entre os construtos, com 1.953 para Planejamento de Compras, 1.956 para Sistema de Avaliação de Fornecedores e 1.648 para Relacionamento comprador-fornecedor. Conforme Cohen et al. (2002) valores acima de 10 indicam sérios problemas de multicolinearidade. Com isso, o modelo apresentou índices de confiabilidade, bem como de validade discriminante, indicando que cada construto é unidimensional.

\section{DIAGRAMA DO MODELO DO PLANEJAMENTO DE COMPRAS E RELACIONAMENTO COMPRADOR E FORNECEDOR}

Os diagramas são a base para a análise de caminhos, os quais calculam a força das relações usando apenas uma matriz de correlação ou covariância como entrada. A correlação simples (bivariada) entre duas variáveis quaisquer pode ser representada como a soma dos caminhos compostos conectando esses pontos (HAIR Jr. et al., 2005).

Para verificar o impacto da estratégia do planejamento de compras no desempenho financeiro da indústria de transformação brasileira, foi realizada a modelagem de equações estruturais (MEE).

Os coeficientes foram padronizados a um valor máximo de 1,0 , sendo que valores próximos a zero têm pouco ou nenhum efeito substantivo e, conforme esse valor aumenta, sua importância intensifica as relações causais (HAIR Jr. et al., 2005). Os resultados serão apresentados conforme as hipóteses elaboradas. Cada figura associará a variável exógena "Planejamento de Compras" a um construto, indicando os parâmetros estimados para o modelo. 
Impacto do planejamento de compras no desempenho financeiro da indústria de transformação do Brasil

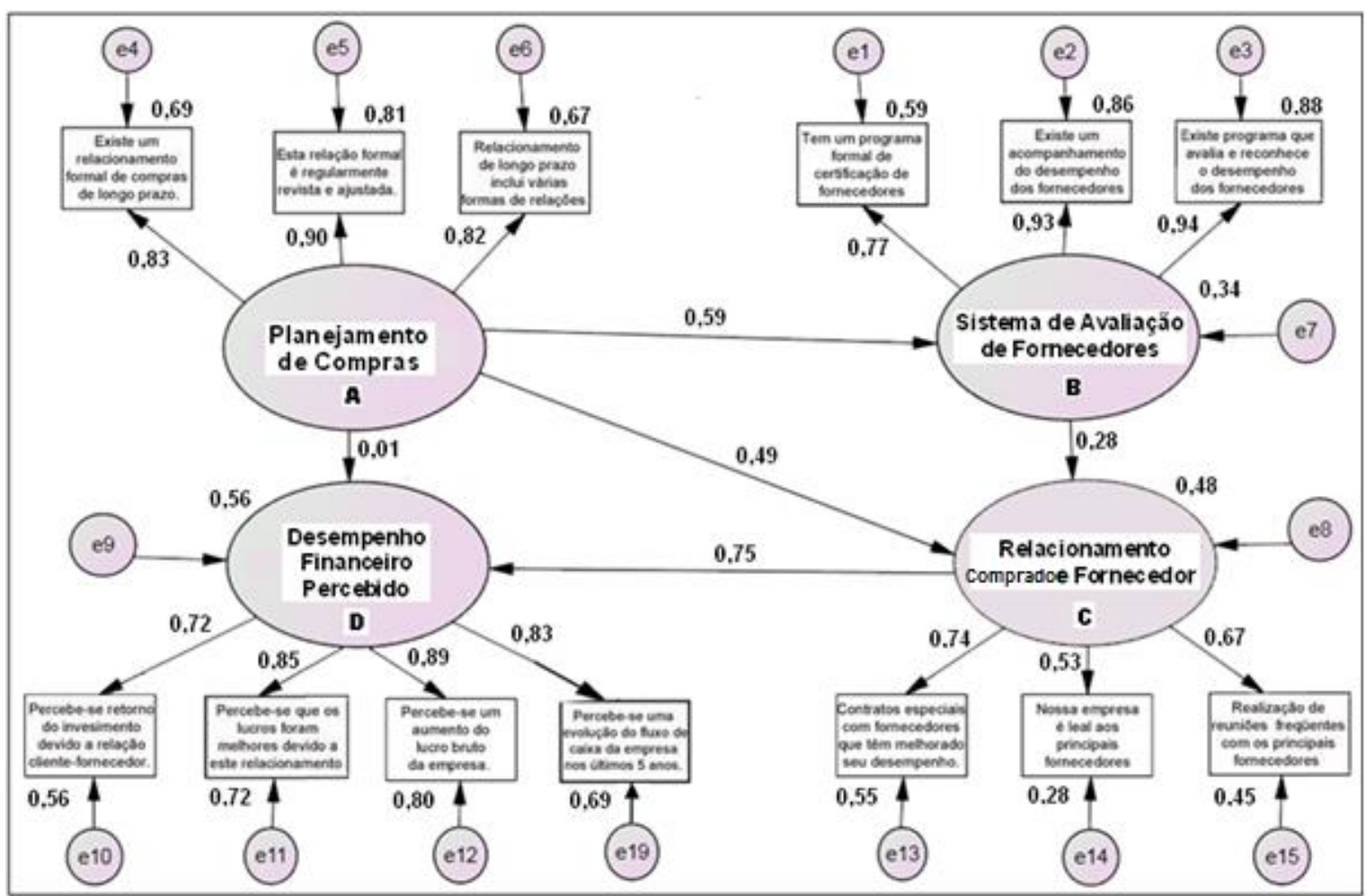

Figura 1 - Modelo do gerenciamento estratégico no Relacionamento entre Comprador -Fornecedor. Fonte: Elaborado pelos autores (2013)

\subsection{H1: O Planejamento de Compras tem impacto positivo sobre o Sistema de Avaliação de Fornecedores}

Inicialmente será avaliada a influência do Planejamento de Compras (A) sobre a dimensão 'Sistema de Avaliação de Fornecedores' (B), conforme a Figura 1, buscando confirmar a primeira hipótese proposta desse estudo.

$\mathrm{O}$ resultado da pesquisa demonstrou um impacto na relação de 'A sobre B' de 0,59 ( $\mathrm{p}<0,05)$, considerado um bom fator de impacto do planejamento de compras sobre o sistema de avaliação de fornecedores confirmando-se a hipótese H1. Para Carr e Pearson (1999) por meio do planejamento de compras os fornecedores poderão ser identificados e qualificados para atender as necessidades da empresa. Os autores indicam ainda que esta avaliação justifica-se também por identificar potenciais fornecedores e determinar suas qualificações.

O planejamento estratégico de compras influência a forma de como os fornecedores serão selecionados e avaliados (SU, 2013). O sistema de avaliação dos fornecedores identifica as ineficiências e estabelece melhorias que auxiliam o fornecedor a compreender as necessidades de seus compradores (PRAHINSKI; BENTON, 2004; TALLURI; 
Luis Cesar Mondini, Denise Del Prá Netto Machado, Marcia Regina Santiago Scarpin \& Vanessa Edy Dagnoni Mondini

NARASIMHAN, 2004). Sobrero e Roberts (2001) ressaltam que quando existe a formalização por meio de contratos, para monitorar a relação entre fornecedores e compradores, os direitos das empresas envolvidas e as fronteiras legais das atividades do processo de desenvolvimento ficam garantidos. Alguns indicadores podem ser considerados chave no setor de compras por possuírem características específicas que os ligam aos fatores relevantes de sucesso do setor e, consequentemente, da organização, daí sua importância estratégica para a empresa.

\subsection{H2: O Planejamento de Compras tem impacto positivo sobre o Relacionamento entre Comprador e Fornecedor}

A seguir, pretende-se averiguar a segunda hipótese (H2), a qual afirma que o Planejamento de Compras (A) possui impacto positivo sobre o 'Relacionamento entre Comprador e Fornecedor' (C).

Conforme Figura 1 constata-se que o relacionamento da área de compras é percebido como relevante para um bom relacionamento e desenvolvimento entre comprador e fornecedor. Na percepção dos respondentes esta relação obteve um impacto de 'A sobre C' de 0,49 ( $\mathrm{p}<0,05$ ), sendo este também, um bom fator de impacto, confirmando-se a hipótese H2.

O planejamento estratégico de compras favorece a relação interorganizacional. A relação como um recurso único pode resultar em vantagem competitiva, por meio da comunicação, compartilhamento de informações e confiança (DYER; SINGH,1998; TERPEND et al., 2008). As relações de cooperação e comunicação entre empresas, compradoras-fornecedoras, geram maior capacidade de resposta ao cliente final (CARR; PEARSON, 1999). O estreitamento da relação estabelecida entre os envolvidos na parceria favorece a coordenação entre as atividades e os processos. Esta proximidade permite a diminuição dos custos que se concentram nas atividades da cadeia por meio da interligação de processos de sistemas computadorizados que irão, consequentemente, melhorar a qualidade e aumentar a velocidade na entrega de produtos/serviços (MAIA; CERRA; ALVES FILHO, 2005; MARTINS; SOUZA, 2010). Além disso, a colaboração entre os participantes da relação precisa de um vínculo dentro da organização que sirva de referência no momento das interações comerciais. Visualizada no modelo pela relação entre A e C, percebe-se que as empresas cada vez mais dependem da interação existente entre os compradores e fornecedores, exigindo que compradores mantenham sólidos relacionamentos com seus fornecedores para ficar à frente da concorrência (MORGAN; HUNT, 1994). 
Impacto do planejamento de compras no desempenho financeiro da indústria de transformação do Brasil

\subsection{H3: O Planejamento de Compras tem impacto positivo sobre o desempenho} Financeiro percebido pela organização.

Neste tópico, almeja-se testar a terceira hipótese, que afirma haver um impacto positivo entre 'Planejamento de Compras' (A) e o 'Desempenho Financeiro percebido' (D). Na percepção dos respondentes esta hipótese (H3) não se confirmou. Na correlação o impacto de 'A para D' foi de apenas 0,01 ( $p>0,10)$, portanto, essa hipótese foi rejeitada.

Ainda que estudos comprovem uma relação positiva entre o planejamento estratégico de compras e o desempenho financeiro (ELLRAM; LIU, 2002), para que ele ocorra dentro das organizações é preciso que o setor de compras seja elevado a um nível estratégico (CARR; SMELTZER, 1997, MENITA et al., 2011). Os processos e práticas que envolvem o planejamento de compras precisam ser bem desenvolvidos, corretamente implantados e controlados, para que impactem positivamente no desempenho financeiro da empresa compradora (BRACKER et al., 1988, CHEN; PAULRAJ; LADO, 2004). Para Porter (1986) existe uma relação entre o nível corporativo estratégico e o nível funcional, o que poderia confirmar esta hipótese, porém conforme Carr e Pearson (1999) esta relação da estratégia corporativa e funcional só é positiva quando corretamente implementada e controlada no processo de planejamento e aplicação da estratégia. Ainda segundo os autores, a função de compras é descrita como parte do processo do planejamento estratégico da empresa, na qual ela avalia, implementa e controla os planos estratégicos operacionais, porém complementam que esta participação deve estar explícita para o setor. Neste sentido, para que o planejamento estratégico de compras impacte positivamente no desempenho financeiro é preciso que o setor de compras considere a aplicação efetiva das práticas e estabeleça uma relação de confiança e uma comunicação efetiva com seus fornecedores (CHENG, YIP \& YEUNG, 2012).

\subsection{H4: O Sistema de Avaliação de Fornecedores tem impacto positivo sobre o}

\section{Relacionamento entre Comprador e Fornecedor.}

Neste item, espera-se verificar a relação do Sistema de Avaliação de Fornecedores (B) sobre o Relacionamento entre Comprador e Fornecedor (C).

O resultado da hipótese testada foi de 0,28 de relevância $(\mathrm{p}<0,05)$, indicando existir um impacto positivo do sistema de avaliação de fornecedores no relacionamento entre compradores e seus fornecedores, confirmando-se a hipótese $\mathrm{H} 4$.

A formalização do sistema de avaliação garante um feedback na relação compradorfornecedor (CARR; PEARSON, 1999; SOBRERO; ROBERTS, 2001; PRAHINSKI; BENTON, 2004), estreita a relação, uma vez que o fornecedor sabe exatamente quais pontos 
Luis Cesar Mondini, Denise Del Prá Netto Machado, Marcia Regina Santiago Scarpin \& Vanessa Edy Dagnoni Mondini

deve melhorar e intensifica a comunicação com fornecedores chave, visando melhores desempenhos e desenvolvimento de competências (SÁNCHEZ-RODRÍGUEZ, 2009; SU, 2013). Uma avaliação eficiente de fornecedores leva a empresa compradora a conhecer os processos e as capacidades de seus fornecedores, integrando-os em relacionamento de longo prazo, com a finalidade de estabelecer um compromisso de prosperidade entre as empresas (LIKER; CHOI, 2004). De acordo com Carr e Pearson (1999) a chave para gerir as relações entre comprador e fornecedor é conhecer os pontos fortes e fracos da relação. Porém, o bom desempenho na avaliação não corresponde necessariamente a negócios futuros entre as partes, mas serve como uma premissa positiva para futuras decisões. Assim, complementam os autores, compradores e fornecedores têm a oportunidade de desenvolver um relacionamento cooperativo, alicerçando um grau de confiança por meio do processo de gestão de fornecedores.

\subsection{H5: Neste item, espera-se verificar a correlação positiva do Relacionamento entre Comprador e Fornecedor (C) sobre o Desempenho Financeiro percebido (D)}

$\mathrm{Na}$ correlação do relacionamento entre comprador e fornecedor verificou-se uma forte relevância sobre o desempenho financeiro percebido pela empresa, conforme a percepção dos respondentes da pesquisa. Confirmando-se a hipótese H5, em que o impacto de 'D sobre C' foi de 0,75 de relevância $(\mathrm{p}<0,05)$. Esse resultado pode ser considerado um fator de impacto relevante e corrobora os estudos de Carr e Perason (1999), nos quais os autores afirmam que o relacionamento entre comprador e fornecedor pode levar a organização a ter um melhor desempenho financeiro.

O relacionamento entre comprador-fornecedor deve ser considerado um fator estratégico na indústria de transformação, pois impacta diretamente no desempenho financeiro da empresa (WATTS; HAHN, 1993, CARR; PEARSON, 1999, CHEN, PAULRAJ; LADO, 2004, CHENG, YIP; YEUNG, 2012). Esta cooperação pode levar ao desenvolvimento de novos produtos, melhorias incrementais, compartilhamento de sistemas de informações, entre outros (SÁNCHEZ-RODRÍGUEZ, 2009, SOUZA; BACIC; BERNARDES, 2009). Conforme Carr e Pearson (1999) o desempenho é um importante indicador de uma empresa, pois sua competitividade está atrelada ao seu desempenho. Contribuem neste aspecto, ainda que em longo prazo, acordos de cooperação entre comprador e fornecedor impactando positivamente sobre o desempenho da empresa (LAIMER; LAIMER, 2009). Para Brito e Brito (2012), em síntese, através das práticas colaborativas podem-se conseguir vantagens de redução dos custos em longo prazo. A escolha correta de indicadores permitirá às parcerias avaliarem o

REAd | Porto Alegre - Edição 80 - N 1 - janeiro/abril 2015 - p. 113-140 
Impacto do planejamento de compras no desempenho financeiro da indústria de transformação do Brasil

desempenho conjunto e identificar ações a serem executadas com base em relacionamentos colaborativos buscando alcançar objetivos da cadeia.

\section{CONSIDERAÇÕES FINAIS}

Este estudo contribui para a literatura do planejamento estratégico de compras e sua relação com o desempenho financeiro, no contexto da indústria de transformação, usando uma pesquisa empírica baseada em uma survey.

A partir da amostra estudada, como principais resultados da estatística descritiva, constatou-se que o planejamento de compras considera a existência de um planejamento formal de longo prazo, se o mesmo é revisto regularmente e se contempla as diversas formas de relacionamento entre comprador e fornecedor. As respostas indicam uma tendência aos relacionamentos duradouros mesmo que pouco formalizados, que incidem sobre a estratégia de longo prazo das organizações pesquisadas. Na análise do sistema de avaliação de fornecedores, percebeu-se que a certificação e o acompanhamento do desempenho já estão bem difundidos no setor. A avaliação deste desempenho, apesar de ter sido em menor percentual, também é observada, indicando um inicio no processo de formalização.

No relacionamento entre comprador e fornecedor, observou-se que os acordos especiais e a fidelidade com fornecedores foram os critérios tidos como mais importantes por parte dos respondentes, seguidos pela influência dos maiores fornecedores e pela comunicação entre a alta direção e os principais fornecedores. Um terceiro bloco de importância poderia ser sinalizado como sendo a presença de reuniões ou encontros e, finalizando, com um percentual mais baixo, a utilização de sistemas de dados para troca de informações. Estes dados sugerem que as relações entre comprador e fornecedor tendem ainda a ocorrer por meio de contratos e contatos 'face-a-face', e menos por sistemas informatizados.

$\mathrm{Na}$ perspectiva do desempenho financeiro percebido, as respostas foram indicativas de que a amostra estudada reconhece o valor do relacionamento entre comprador e fornecedor. Todos os resultados desta dimensão apontam para a existência do impacto positivo deste relacionamento nos resultados das empresas.

Conforme o modelo proposto na pesquisa as Hipóteses $\mathrm{H} 1, \mathrm{H} 2, \mathrm{H} 4$ e H5 confirmaram-se positivas e a H3 - Planejamento de Compras sobre o Desempenho Financeiro percebido foi rejeitada. A hipótese rejeitada sugere que os respondentes não consideram que o planejamento de compras gera impactos diretos no desempenho da empresa, ou não visualizam a configuração desta relação, porém, consideram importantes as relações 
Luis Cesar Mondini, Denise Del Prá Netto Machado, Marcia Regina Santiago Scarpin \& Vanessa Edy Dagnoni Mondini

observadas nas hipóteses $\mathrm{H} 1$ e H2, percebendo-se a relevância das práticas que envolvem o planejamento de compras para o alcance de um melhor desempenho financeiro. Além disso, reforça os estudos de Yang et al. (2013) de que o planejamento estratégico de compras deve levar em conta a cultura do país em que as empresas estão instaladas. Pelo resultado da pesquisa, no Brasil, a relação entre planejamento de compras e desempenho financeiro só é percebida pelas indústrias de transformação, quando as práticas de sistema de avaliação do fornecedor e relacionamento comprador-fornecedor são consideradas, indicando esta ser uma relação indireta.

Os resultados desta pesquisa indicam a relevância da gestão de suprimentos para as empresas que compõem a indústria de transformação, podendo auxiliar na estruturação de modelos de gestão para esta área. Pode-se, também, indicar a utilização deste modelo para análise de outros ramos industriais, que poderão, além de fomentar a área acadêmica, auxiliar nas relações comprador/fornecedor.

De acordo com as relações dos construtos apresentados pode-se afirmar que o planejamento de compras fortalece as ações da estratégia empresarial operacionalizada pela área de compras quando procura fomentar a organização com bons fornecedores. Assim, este estudo, levando em consideração o planejamento de compras estrategicamente alinhado e convergindo com os objetivos gerais da empresa, confirma que, a manutenção do relacionamento estruturado com fornecedores potencialmente desenvolvidos, no longo prazo, pode incorrer em resultados favoráveis no desempenho financeiro organizacional, ampliando a vantagem competitiva das empresas estabelecidas na cadeia de valor.

Todo método tem limitações. Devido ao fato da amostragem ter sido por conveniência os resultados da pesquisa não devem ser extrapolados para um universo maior do que as empresas pesquisadas. Outro fator a ser considerado é o universo dos respondentes, que, em sua maioria, atuam como empregados de nível tático e operacional das organizações. Neste nível, nem sempre se possui acesso efetivo aos balanços financeiros, demonstrativos de resultado e aos índices de evolução financeira das empresas no período de cinco anos conforme solicitavam as perguntas da dimensão quatro desta pesquisa. Além disso, as perguntas sobre desempenho financeiro foram realizadas considerando a percepção dos respondentes e não dados absolutos.

Para futuras pesquisas e complementação deste trabalho sugere-se que seja realizada uma pesquisa direcionada somente ao nível gerencial estratégico das empresas. Pode-se ainda, realizar uma pesquisa direcionada a um setor específico da indústria de transformação e, buscar maior validação do construto financeiro por meio de dados absolutos, que poderão ser

REAd | Porto Alegre - Edição 80 - N 1 - janeiro/abril 2015 - p. 113-140 
Impacto do planejamento de compras no desempenho financeiro da indústria de transformação do Brasil

adquiridos, além da percepção dos respondentes, em bases financeiras. Além disso, recomenda-se um estudo de caso longitudinal para avaliação das percepções ao longo do tempo.

\section{REFERÊNCIAS}

BNDeS. Classificação das empresas. Disponível em http://www.bndes.gov.br/SiteBNDES/bndes/ bndes_pt/Institucional/acesso_a_informacao/. Acesso em 20 set. 2012.

BRACKER, J. S.; KEATS, B. W.; PEARSON, J. N. Planning and financial performance among small firms in a growth industry. Strategic Management Journal, v.9, n.6, p.591603, 1988.

BRITO, R. P. de; BRITO, L. A. L. Vantagem competitiva e sua relação com o desempenho. RAC - Revista de Administração Contemporânea, Rio de Janeiro, v.16, n.3, art. 2, p.360380, Maio/Jun. 2012.

BRITO, L. A. L.; BRITO, E. P. Z.; HASHIBA, L. H. What type of cooperation with suppliers and customers leads to superior performance? Journal of Business Research, v.67, n.5, p.952-959, 2014.

CARR, A. S.; PEARSON, J. N. Strategically managed buyer-supplier relationships and performance outcomes. Journal of Operations Management, v.17, p.497-519, 1999.

CARR, A. S.; PEARSON, J. N. The impact of purchasing and supplier involvement on strategic purchasing and its impact on firm's performance. International Journal of Operations \& Production Management, v.22, n.9, p.1032-1053, 2002.

CARR, A.S., SMELTZER, L.R. An empirically based operational definition of strategic purchasing. European Journal of Purchasing and Supply Management, v.3, n.4, p.199207, 1997.

CARTER, C. R.; KALE, R.; GRIMM, C. M. Environmental purchasing and firm performance: an empirical investigation. Transportation Research Part E: Logistics and Transportation Review, v.36, n.3, p.219-228, 2000.

CHEN, I. J.; PAULRAJ, A. Towards a theory of supply chain management: the constructs and measurements. Journal of Operations Management, v.22, n.2, p.119-150, 2004. 
Luis Cesar Mondini, Denise Del Prá Netto Machado, Marcia Regina Santiago Scarpin \& Vanessa Edy Dagnoni Mondini

CHEN, I. J.; PAULRAJ, A.; LADO, A. A. Strategic purchasing, supply management, and firm performance. Journal of Operations Management, v.22, n.5, p.505-523, 2004.

CHENG, T. C. E.; YIP, F. K.; YEUNG, A. C. L. Supply risk management via guanxi in the chinese business context: the buyer's perspective. International Journal of Production Economics, v.139, n.1, p.3-13, 2012.

CHIANG, C.; KOCABASOGLU-HILLMER, C.; SURESH, N. An empirical investigation of the impact of strategic sourcing and flexibility on firm's supply chain agility. International Journal of Operations \& Production Management, v.32, n.1, p.49-78, 2012.

COHEN, M.; SILVA, J. F. O Impacto das decisões estratégicas no desempenho dos franqueados em fast-food: o papel do relacionamento franqueador-franqueado. Revista de Administração Contemporânea. v.4, n.2, 2000.

DAS, A.; NARASIMHAN, R. Purchasing competence and its relationship with manufacturing performance. Journal of Supply Chain Management, v.36, n.1, p.17-28, 2000 .

DYER, J. H.; SINGH, H. The relational view: cooperative strategy and sources of interorganizational competitive advantage. Academy of Management Review, v.23, n.4, p.660-679, 1998.

ELLRAM, L. M.; LIU, B. The financial impact of supply management. Supply Chain Management Review, v.6, n.6, p.30-37, 2002.

FORNELL, C.; LARCKER, D. F. Evaluating structural equation models with unobservable variables and measurement error. Journal of Marketing Research, v.18, p.39-50, 1981.

GUERRA, J. H. L.. Análise da restrição da oferta de insumos básicos em períodos econômicos favoráveis da perspectiva de um fabricante de avião. REAd Revista Eletrônica Administração, v.20, n.2, p.571-600, 2014 .

HAIR Jr., J. F.; BABIN, B.; MONEY, A. H.; SAMOUEL, P. Fundamentos e métodos de pesquisa em administração. Porto Alegre: Bookman, 2005.

HALD, K. S.; ELLEGAARD, C. Supplier evaluation processes: the shaping and reshaping of supplier performance. International Journal of Operations \& Production Management, v.31, n.8, p.888-910, 2011. 
Impacto do planejamento de compras no desempenho financeiro da indústria de transformação do Brasil

HANSEN, M. T.; NOHRIA, N. How to build collaborative advantage. MIT Sloan Management Review, v.46, n.1, p.22-30, 2004.

IBGE. Indicadores conjunturais. Disponível em http://www.ibge.gov.br/home/estatistica/pesquisas/indicadores.php. Acesso em 16/11/2014.

JORGE, M. J. ; AVELlAR, C. M.; MELO, L. C.; PIGATTO, J. A. M.; BATISTA, D. L. Indicadores de Efetividade em custo de atividades-meio no modelo de gestão para resultados: a experiência do Ipec/Fiocruz. Repec - Revista de Educação e Pesquisa em Contabilidade. v.4, n.2, p.1-22, 2010.

KOCABASOGLU, C.; SURESH, N. C. Strategic sourcing: an empirical investigation of the concept and its practices in US manufacturing firms. Journal of Supply Chain Management, v.42, n.2, p.4-16, 2006.

LAIMER, C. G.; LAIMER, V. R. (2009). Relações de cooperação na perspectiva da visão baseada em recursos. Revista de Administração da Unimep, v.7, n.3, p.93-110.

LARSON, P.D. An empirical study of inter-organizational functional integration and total costs. Journal of Business Logistics, v.15, n.1, p.153-169. 1994.

LIKER, J. K.; CHOI, T. Y. Building Deep Supplier Relationships. Harvard Business Review, v.82, n.12, p.104-113, december, 2004.

MAIA, J. L.; CERRA, A. L.; ALVES FILHO, A. G. Inter-relações entre estratégia de operações e gestão da cadeia de suprimentos: estudos de caso no segmento de motores para automóveis. Gestão de Produção, v.12, n. 3, p. 377-391, 2005.

MARTINS, R.; SOUZA, O. V. Análise do alinhamento de práticas colaborativas entre camadas: estudo comparativo de casos nas cadeias automobilísticas de São Paulo e de Minas Gerais. Revista de Administração da UNIMEP, v.8, n.3, Setembro / Dezembro, 2010.

MCHUG, M.; HUMPHREYS, P.; MCIVOR, R. Buyer-Supplier relationships and organizational health. The Journal of Supply Chain Management, p.15-25, May, 2003.

MENITA, P. R.; VANALlE, R. M.; SALlES, J. A. A.; OliVEIRA, R. D. Análise das estruturas de governança como instrumento de gestão de compras estratégicas. Revista Científica Indexada Linkania Master, v.1, n. 1, 2011. 
Luis Cesar Mondini, Denise Del Prá Netto Machado, Marcia Regina Santiago Scarpin \& Vanessa Edy Dagnoni Mondini

MERLO, E. M.; CERIBELI, H. B.; PRADO, L. S. Gestão de perdas no pequeno varejo: um estudo de caso de uma rede de compras formada por pequenos supermercadistas. Revista de Administração da UNIMEP. v.9, n.3, Setembro/Dezembro, 2011.

MOLLER, M. M.; JOHANSEN, J.; BOER, H. Managing buyer-supplier relationships and inter-organisational competence development. Integrated Manufactoring Systems, v.14, n.4, p.369-379, 2003.

MOREIRA, M. F.; VARGAS, E. R. Compras para a inovação: casos de inovações induzidas por clientes públicos. RAM - Revista Administração Mackenzie. v.13, n.5, p.232-257, 2012.

MORGAN, R. M.; HUNT, S. D. The Commitment-trust theory of relationship marketing. Journal of Marketing, v.58, n.3, p.20-38, 1994.

NARASIMHAN, R.; DAS, A. The impact of purchasing integration and practices on manufacturing performance. Journal of Operations Management, v.19, n.5, p.593-609, 2001.

NEI. Noticiário de Equipamentos Industriais. Disponível em http://www.nei.com.br/MidiaKit/CatalogoOnline. Acesso em: fevereiro de 2012.

PEREIRA, R. C. F.; LUCE, F. B. Até que os custos os separem: por que clientes e fornecedores estabelecem práticas relacionais para o desenvolvimento conjunto de projetos de software? RAC - Revista de Administração Contemporânea, Curitiba, v.13, n.4, art. 2, p.545-563, Out./Dez. 2009.

PETERSEN, K.J.; HANDFIELD, R.B. ; RAGATZ, G.L. Supplier integration into new product development: coordinating product, process and supply chain design. Journal of Operations Management. v.23, p.371-388, 2005.

PORTER, M. E. Estratégia competitiva: técnicas para análise de indústrias e da concorrência. Rio de Janeiro: Campus, 1986.

PRAHINSKI, C.; BENTON, W. C. Supplier evaluations: communication strategies to improve supplier performance. Journal of Operations Management, v.22, n.1, p.39-62, 2004.

SÁNCHEZ-RODRÍGUEZ, C. Effect of strategic purchasing on supplier development and performance: a structural model. Journal of Business \& Industrial Marketing, v.24, n.3/4, p.161-172, 2009. 
Impacto do planejamento de compras no desempenho financeiro da indústria de transformação do Brasil

SEBRAE. Classificação de empresas. Disponível em <http://www.sebraesc.com.br/leis/default.asp?vcdtexto=4154.>. Acesso em 27 fev. 2013.

SOBRERO, M.; ROBERTS, E. B. The trade-off between efficiency and learning in interorganizational relationships for product development. Management Science, v.47, n.4, apr, 2001.

SOUZA, M. C. A. F.; BACIC, M. J.; BERNARDES, J. M. R. A gestão estratégica das compras como política para reduzir custos. Gestão \& Regionalidade, v.25, n.74, Mai/Ago, 2009.

SU, J. Strategic sourcing in the textile and apparel industry. Industrial Management \& Data Systems, v.113, n.1, p.23-38, 2013.

TALLURI, S.; NARASIMHAN, R. A methodology for strategic sourcing. European journal of operational research, v.154, n.1, p.236-250, 2004.

TERPEND, R.; TYLER, B. B.; KRAUSE, D. R.; HANDFIELD, R. B. Buyer-supplier relationships: derived value over two decades. Journal of Supply Chain Management, v.44, n.2, p.28-55, 2008.

VENKATRAMAN, N.; RAMANUJAM, V. Measurement of business performance in strategy research: a comparison of approaches. Academy of Management Review, v.11, n.4, p.801-814, 1986.

WATTS, C.; HAHN, C. Supplier development programs: an empirical analysis. International Journal of Purchasing and Materials Management, v.29, n.2, p.10-17, 1993.

WEBER, C.A.; CURRENT, J. \& BENTON, W.C. Vendor selection criteria and methods. European Journal of Operational Research. v.50, p.2-18, 1991

YANG, C.; LIN, R.; KRUMWIEDE, D.; STICKEL, E.; SHEU, C. Efficacy of purchasing activities and strategic involvement: an international comparison. International Journal of Operations \& Production Management, v.33, n.1, p.49-68, 2013. 